\title{
Keynote Lecture
}

\section{KN16}

Properties of two dimensional materials obtained from experiments in a low-voltage aberration-corrected TEM

\author{
$\underline{\text { U. Kaiser }}^{1}$ \\ ${ }^{1}$ Ulm University, Group of Electron Microscopy for Materials Science, Ulm, Germany
}

We report on structural and electronic properties of two-dimensional materials ob-tained by analytical low-voltage aberrationcorrected transmission electron microscopy. Basic crystallographic defects and their peculiarities will be discussed for twodimensional materials at the atomic level. Thus, we report the atomic structure of point defect and -clusters [1], the full life circle of dislocations [2] and the movements of grain boundaries in grapheme [3]. In addition, we unravel the atomic structure of the amorphous phase (graphene, $\mathrm{SiO}_{2}$ ) in direct space just from single-atom-based analysis of high-resolution TEM images [5, 6]. As the energetic electron beam is interacting with the specimen via transferring energy to the atoms, structural transformation between different phases can be followed atom-by-atom [7, 8, 9]. In addition, physical properties such as the knock-on damage threshold is determined from controlled direct space experiments and precise measurements of high-resolution TEM images of graphene and $\mathrm{MoS}_{2}[8,7]$. However beam-electron interactions with the specimen are also restricting imaging the pristine structure of a sample. It can be suppressed by simply limiting the total electron doses on the samples. Limited electron doses, however, result in worse signal to noise ratios. Here, a quantitative approach for estimating the visibility of objects in TEM images with limited doses will be presented [10]. Another traditional approach to suppress electron-induced damage during HRTEM observation is to employ an efficient cleaning procedure [11] and the protective coating of sensitive materials. This old approach will be taken to its extreme, when radiation sensitive materials are enclosed inside carbon nanotubes [12] and between two graphene layers [13]. We show moreover the advantage of lowering the accelerating voltage for imaging the pristine structure of low-dimensional materials [14]. [4] P. Wachsmuth, R. Hambach, M.K. Kinyanjui, et al., Phys. Rev. B B 88, 075433, (2013) [5] P. Y. Huang, S. Kurasch, A. Srivastava, et al. Nano Lett. 12(2), 1081, (2012) [6] P. Y. Huang, S. Kurasch, J.S. Alden, et al., Science 342, 224, (2013) [7] H.-P. Komsa, J. Kotakoski, S. Kurasch, et al., Phys. Rev. Lett. 109, 035503 (2012) [8] C Meyer, F Eder, S Kurasch, et al. Physical Review Letters, 108, 196102. 2012. [9] B. Westenfelder, J. C. Meyer, J. Biskupek, et al., Transformations of Carbon Adsorbates on Graphene Substrates under Extreme Heat, Nano Letters, 11 (12), 5123-5127, 2011 [10] Z. Lee, H. Rose, O. Lehtinen, et al., Ultramicroscopy (2014), DOI 10.1016/j.ultramic.2014.01.010 [11] G. Algara-Siller, S. Kurasch, M. Sedighi, et al., Appl. Phys. Lett. 103 (2013) 203107 [12] T. Zoberbier, T. W. Chamberlain, J. Biskupek, et al., J. Am. Chem. Soc. 134 (2012) 3073-3079 [13] G. Algara-Siller, S. Kurasch, M. Sedighi, et al., Appl. Phys. Lett. 103. 203107, (2013) [14] U. Kaiser et al. Ultramicroscopy, 111, 8, 1239, (2011) [15] Fruitful cooperation within the SALVE project and financial support by the DFG (German Research Foundation) and by the Ministry of Science, Research, and the Arts (MWK) of Baden-Württemberg are gratefully acknowledged.

[1] J. Kotakoski, J. Meyer, S. Kurasch, et al. Physical Review B, 83 (2011), [2] O. Lehtinen, S. Kurasch, A.V. Krasheninnikov et al. Nature Communications 4, 3098, (2013), [3] S. Kurasch, J. Kotakoski, O. Lehtinen, et al. Nano Lett. 12 (6), 3168, (2012)

Keywords: low-voltage transmission electron microscopy, crystallographic defects in two-dimensional materials, amorphous phase 